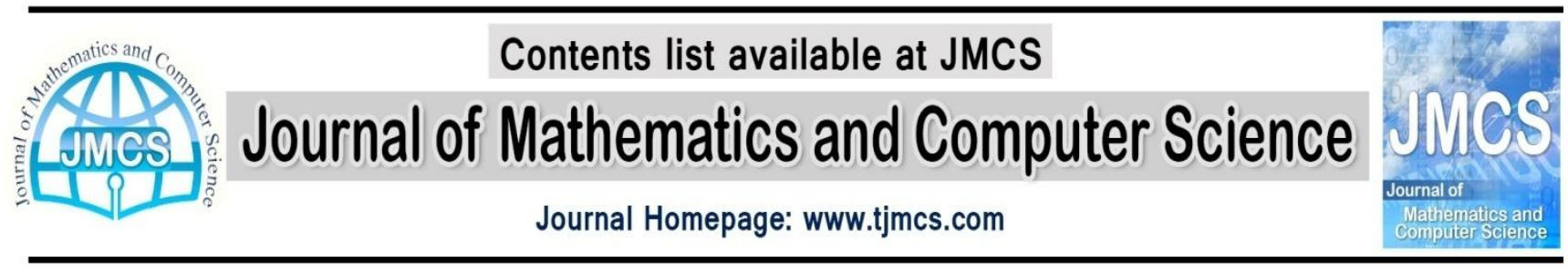

\title{
Application of Unsharp Mask in Augmenting the Quality of Extracted Watermark in Spatial Domain Watermarking \\ Saeed Amirgholipour ${ }^{1 *}$,Ahmad Reza Naghsh-Nilchi ${ }^{2}$,Aboosaleh Mohammad Sharifi ${ }^{1}$, Mehdi Alirezanejad ${ }^{3}$, Moien $\mathrm{Arab}^{4}$ \\ ${ }^{1}$ Department of Computer, Islamic Azad University, Ramsar Branch, Ramsar, Iran \\ ${ }^{2}$ Department of Computer Engineering, University of Isfahan, Isfahan, Iran \\ ${ }^{3}$ Department of Computer, Islamic Azad University, Firouzkouh Branch, Firouzkouh, Iran \\ ${ }^{4}$ Department of Computer, Sharif University of Technology International Campus, Kish, Iran
}

Article history:

Received May 2014

Accepted June 2014

Available online June 2014

\section{s.kasmani@iauramsar.ac.ir}

\begin{abstract}
It is generally believed that spatial domain watermarking methods are not robust against common image processing attacks. In this paper, a novel application of an unsharp mask is proposed to increase the performance of watermark recovery in correlation based watermarking. Commonly, unsharp mask is used to improve quality of the image by emphasizing its high frequency contents to enhance the edge and details information in it. Dissimilarity between different parts of image is increased by this filter. Thus, the watermark could recover more correctly from the watermarked image. This filter is applied before running watermark recovery procedure. To show efficiency of proposed filter on the spatial domain watermarking, it is applied on a typical correlation based method. Several experiments are done to evaluate the performance of proposed method is improved by applying this filter before watermark extraction procedure.
\end{abstract}

Keywords: Unsharp Mask, Digital Image Watermarking, Correlation Based Watermarking, Spatial, Domain Watermarking

\section{Introduction}

The watermarking has been proposed as an appropriate solution for copyright protection methods for digital data. The watermarking methods embed a watermark with some extra information about the digital media without visibly altering them [1]. Digital watermarking methods are consisting of two parts; embedding and recovery algorithms. In the embedding algorithm, the watermark is embedded in digital media with a special algorithm. And this watermark is recovered from the watermarked image by extracting algorithm. 
Robustness and imperceptibility are two important factors in watermarking algorithm. But these properties are incompatible with each other. The imperceptibility means that how much the media quality is changed by the embedded watermark; and the robustness is the ability to recover the watermark after common or malicious signal processing operations. These incompatible parameters should be set to meet the requirements of the application [2], [3].

Generally, there are two common domain that watermark is embed in them; the spatial domain or the transform domain. The watermark is embedded straight forward in the pixels of the host image in the spatial domain methods. But, these methods are not robust to image common image processing operation [4]. Although, some paper used the filters for increasing the power of recovery algorithm, [6]. Transform domain watermarking methods take advantage of the transform domain properties to embed the watermark. These methods usually use the Discrete Cosine Transform (DCT) [7], [8] and the Discrete Wavelet Transform (DWT) [9], [10]. These methods typically are more imperceptible and robustness to common image processing operation.

Some researchers have tried to increase the power of watermarking algorithms by augmenting the watermark retrieval algorithms. They try to make watermark information more visible to the recovery algorithms. The goal of these methods is to boost watermark detection rate by an efficient and simple model based on image enhancement, edge detection and noise magnifying methods [5].

A slightly more computationally complex detection scheme is proposed which accompanied by a fixed threshold that performs better than those existing in bibliography [11]. The choice of the transform used, the embedding parameters or the attacks used, do not affect its performance.

Application of blurring filter for increasing the possibility of detection of watermark is proposed by Broadway [12]. They use blurring filters for a watermark with a dominant low frequency content to improve the detection probability. Since the content of image might interfere with the watermark, especially in the low-frequency parts, the reliability of the detector could be improved by applying matched filtering before correlation [6]. This decreases the influence of the original image to the correlation. Therefore, the watermark could easily be recovered from watermarked image.

Hafiz proposed an approach to blind watermark detection for spread spectrum by using of Independent Component Analysis theory [13]. It uses the theory of independent component analysis (ICA) and detects the watermark with a blind source separation method. The watermark information is considered as noise for the watermarked image in its spatial domain. This noise is magnified before executing recovery algorithm, and then recovers the watermark information by adjusting the extracted data from the frequency domain according to the global minimum method [14]. A preprocessing method is proposed that exploit a combination of noise boosting and filtering to facilitate recovering the watermark from watermarked image in the DCT-based watermarking algorithm [3], [5].

In this paper, a novel application of unsharp mask is presented to augment power of recovery algorithms in the spatial domain methods. The dissimilarity between the watermark and the original image are amplified by this filter. Thus, the watermark could be removed from the modified watermarked image meaningfully better. This filter is applied before running watermark extraction procedures in correlation based method. Several experiments are done to evaluate the efficiency of unsharp mask in the spatial domain based watermarking.

The rest of the paper is organized as follows. In section 2, unsharp mask is described in details. A typical correlation based method is introduced as a representative of spatial domain watermarking in section 3. Section 4 presents the experimental results to show the performance of this scheme on the classical method. Conclusions are drawn in Section 5.

\section{Materials and Method}




\subsection{Unsharp Mask}

In this paper, Gonzales definition is used for unsharp masking [15].Linear unsharp masking is a method for augmenting the perceptual quality of an image by highlighting its high-frequency components. In this filter, a high pass filtered of the input signal is added to the original signal to gain the enhanced image [16].

In the linear UM algorithm, the unsharp image funsharp $(x, y)$ is obtained from the input image $f(x, y)$ as

$f_{\text {unsharp }}(x, y)=\lambda * H P(x, y)+f(x, y)$

Where $\operatorname{HP}(x, y)$ is a linear high pass filter, and $\lambda$ is an appropriate positive scaling factor which controls the contrast enhancement at the output.

The unsharp masking process is shown in the Figure 1.

In this paper, a typical high pass filter is used for Unsharp Mask. It is shown in the $3 \times 3$ convolution matrix looks as follows:

$\left[\begin{array}{ccc}-1 & -2 & -1 \\ -2 & 12 & -2 \\ -1 & -2 & -1\end{array}\right]$

\subsection{Correlation Based Watermarking}

\subsubsection{Watermark Embedding Algorithm}

The watermark embedding process is shown in Figure. 2, and it is describedin the following steps.

* Divide the host image into $8 \times 8$ blocks.

* Re-formulate the watermark image into a vector of zeroes and ones.

* Generate two uncorrelated pseudorandom sequences by a key. One sequence is used to embed the watermark bit 0 (PN_0) and the other sequence is used to embed the watermark bit 1 (PN_1). Number of elements in each of the two pseudorandom sequences must be equal to the number of block.

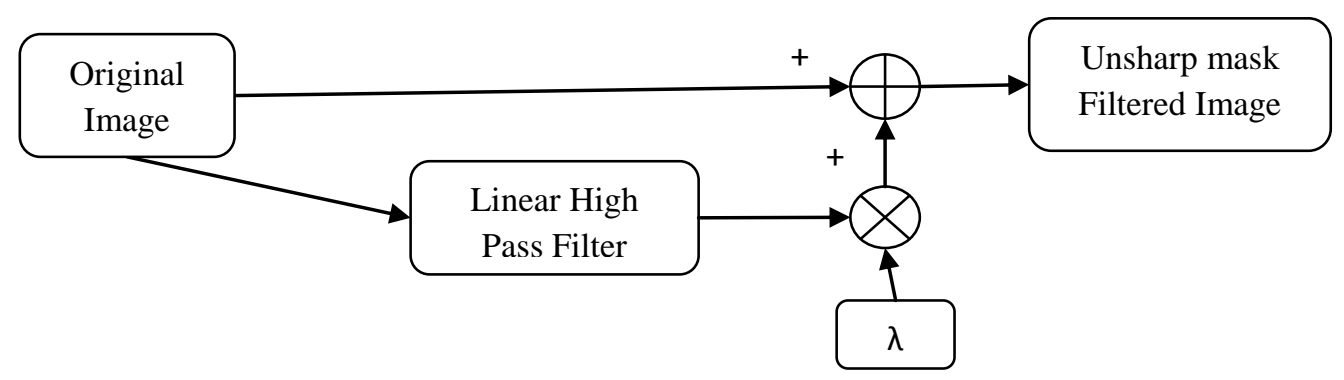

Figure 1: Unsharp masking process 


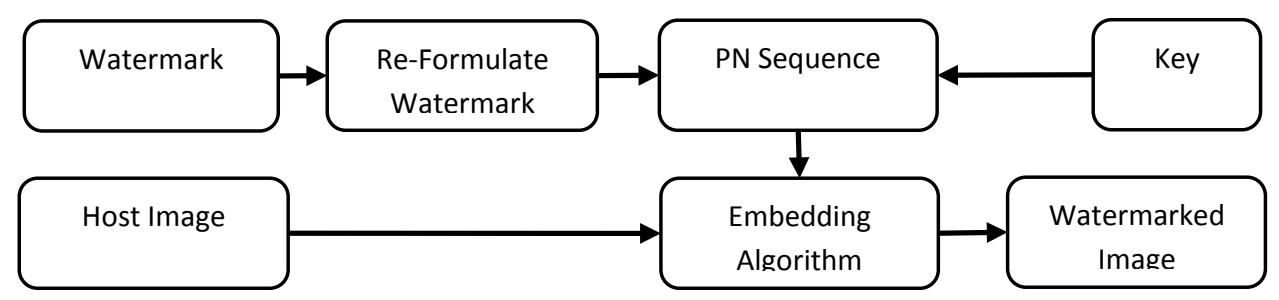

Figure 2: The watermark embedding process

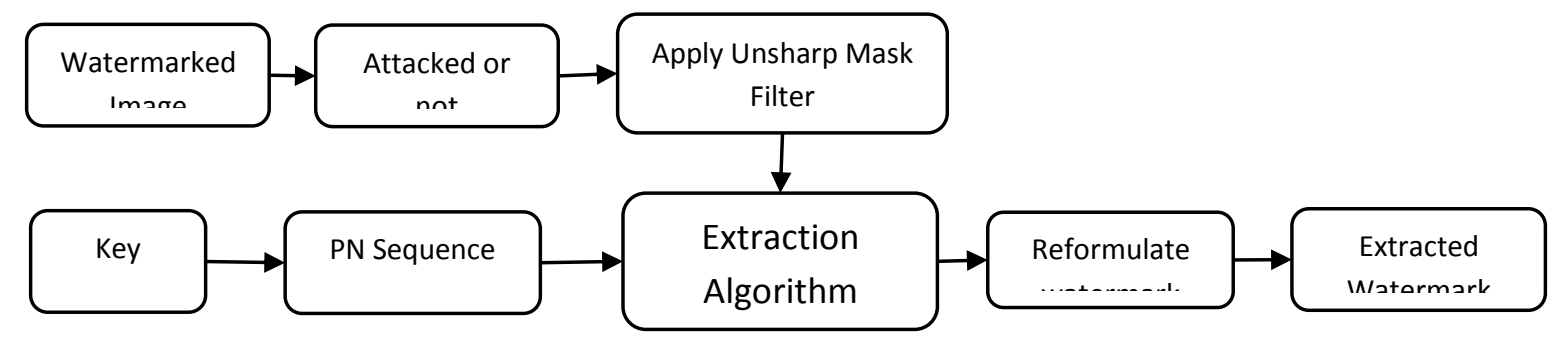

Figure3: The watermark extracting with applying Unsharp Mask before preforming extracting algorithm.

* Embed the two pseudorandom sequences, PN_0 and PN_1, with a gain factor $\alpha$ in the $16 \times 16$ blocks of the host image. If we donate $X$ as the matrix of the block, then embedding is done as equation (3):

$$
X^{\prime}= \begin{cases}X+\alpha^{*} P N 0 & \text { watermark_bit }=0 \\ X+\alpha^{*} P N 1 & \text { watermark_bit }=1\end{cases}
$$

\subsubsection{WatermarkExtracting Algorithm}

The typical correlation based algorithm is a blind watermarking algorithm, and thus the original host image is not required to extract the watermark. Recovery method is the same as embedding one, and filtering is convolved before running extraction and before calculates the correlation on each block to better separate watermark information from host image. The watermark extraction procedure is shown in Figure. 3, and described in details in the following steps.

* Applying Unsharp Mask filter as shown in the equation of (1) on the watermarked image.

* Divide watermarked image that could be attacked or not into $8 \times 8$ blocks.

* Regenerate the two pseudorandom sequences (PN_0 and PN_1) using the same key which used in the watermark embedding procedure.

* For each block in the watermarked image calculate the correlation between the element and the two generated pseudorandom sequences (PN_0 and PN_1). If the correlation with the PN_0 was higher than the correlation with PN_1, then the extracted watermark bit is considered 0 , otherwise the extracted watermark is considered 1.

* The scrambled watermark is reconstructed using the extracted watermark bits. 


\section{Experimental Results}

To compare the efficiency of the proposed filter on correlation based methods, three standard grayscale images with different contents of size $512 \times 512$ are used in our experiments, as shown in Figure. 4. (a)-(c). Pepper is used as a representation of image with low spatial frequency and Barbara as a representation of image with average spatial frequency and Baboon as a representation of image with high spatial frequency. In this experiment, a $32 \times 32$ binary image, as shown in Figure. 4.(d) is taken as the watermark of images. The effect of the Unsharp Mask filters is investigated by measuring imperceptible and robustness of watermarked image. For the imperceptible capability, a quantitative index, Peak Signal-to-Noise Ratio (PSNR), is employed to evaluate the difference between an original image $\mathrm{O}$ and a watermarked image. For the robust capability, Normalized CrossCorrelation (NC) measures the difference between an original watermark W and the corresponding extracted one. If a method has higher NC, it is more robust. The PSNR and the NC are, respectively, defined by equation 4 and 5; respectively,

$$
\begin{aligned}
& \operatorname{PSNR}(\mathrm{O}, \overline{\mathrm{O}})=10 \log _{10} \frac{255 \times 255}{\frac{\left.\sum_{\mathrm{i}=0 \mathrm{j}=0}^{\mathrm{I}-1 \mathrm{~J}-1}\|\|_{\mathrm{ij}}-\overline{\mathrm{O}}_{\mathrm{ij}} \|\right)^{2}}{\mathrm{I} \times \mathrm{J}}} \\
& N C=\frac{\sum_{i=1}^{M_{1}} \sum_{j=1}^{M_{2}} W(\mathrm{i}, \mathrm{j}) \cdot \mathrm{W}^{\prime}(\mathrm{i}, \mathrm{j})}{\sqrt{\sum_{i=1}^{M_{1}} \sum_{j=1}^{M_{2}}[W(\mathrm{i}, \mathrm{j})]^{2}} \sqrt{\sum_{i=1}^{M_{1}} \sum_{j=1}^{M_{2}}\left[W^{\prime}(\mathrm{i}, \mathrm{j})\right]^{2}}}
\end{aligned}
$$

The watermarked image $\mathrm{O}$ is obtained following the completion of the watermark embedding procedure. The watermark information is embedded with PSNR 30, 35, and $40 \mathrm{~dB}$ in the watermarked images. Then Unsharp Mask filter which is described in Section 2 are performed on these watermarked images which may be attached by the method presented in section 3. NC between the original $\mathrm{W}$ and the extracted watermark $\mathrm{W}^{\prime}$ is calculated for different PSNR. To compare the effect of different places of preforming proposed filter, the algorithm is tested byseveral attacks, including JPEG compression, image scaling, adding Salt and Pepper noise, Gaussian filtering. The presented method is implemented using MATLAB.

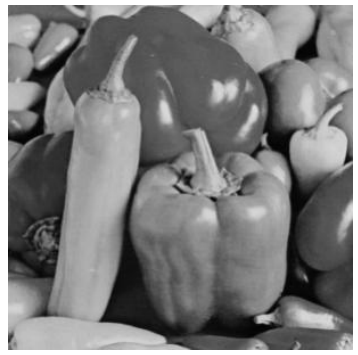

(a)

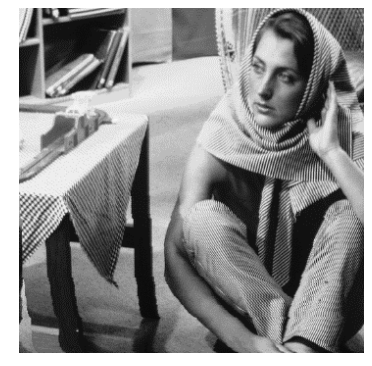

(b)

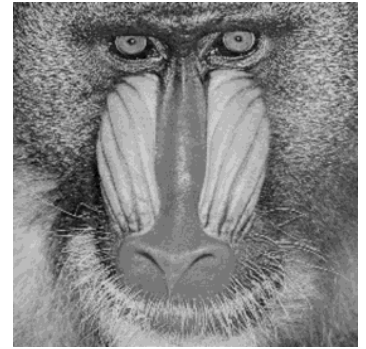

(c)

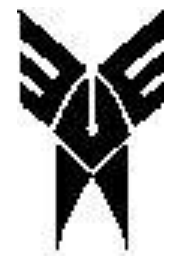

(d)

Figure 4: (a) The original Pepper image; (b) the original Barbara image; (c) the original Baboon image; (d) the original watermark. 
Table 1. Visual comparison between the extracted watermark from the peppers watermarked image with PSNR= 30.

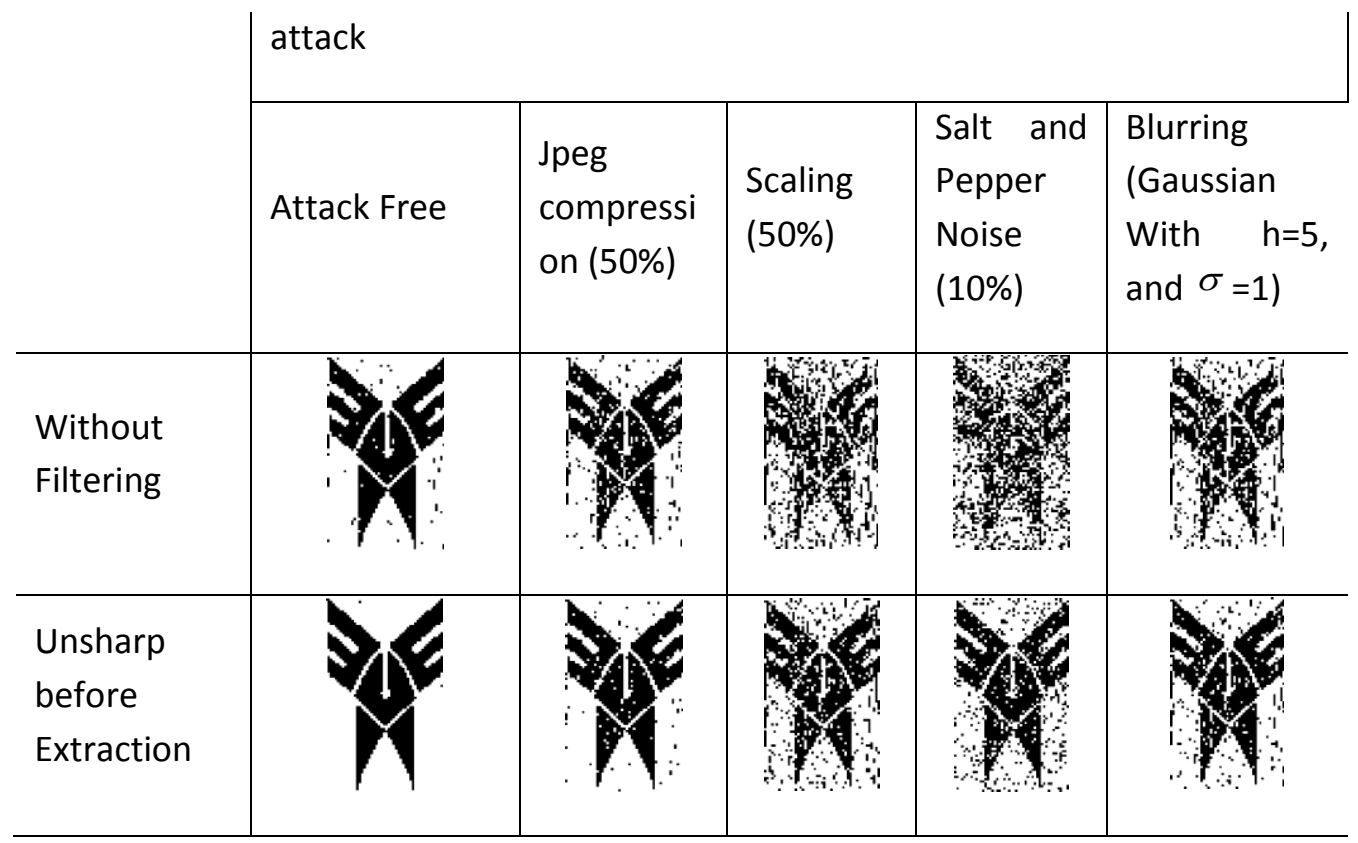

\subsection{Visual Comparisons}

Table 1 visually shows the effects of using unsharp mask filter on extracted watermark in the correlation based watermarked image. These results have been gain form Pepper's imagewhich watermarked with PSNR 30. As shown in the table 1, convolving the Unsharp Mask filter before running extraction procedure significantly improve the quality of extracted watermark.

\subsection{Robustness Comparison}

In the second experiment, the results of preforming Unsharp Mask filter on Pepper, Barbara, Baboon images as a representation of image with low, average, and high spatial frequencies are presented. The goal of this section is to show the effect of convolving this filter on different watermarked image. These results are shown in the table 2-4 for the Pepper, Barbara, Baboon images respectively. As it is shown in the table 2, results are meaningfully improved by exploiting proposed filter in compare with normal recovery method. Utilizing proposed filter before recovery algorithm is more effective to the attacked images with salt and pepper noise, scaling and blurring attacks. However, in the jpeg compression attacks, convolving Unsharp Mask filter increases the results. As it shown in this table, improvement in the values of NC, in salt and pepper noise, scaling and blurring attacks on pepper image with PSNR 40 as a high invisible watermarked image, NC preforms 0.10 much better than normal recovery, and in the case of jpeg compression attack improvement is 0.05 in term of NC. Therefore, for low spatial frequency, using the Unsharp Mask before running extraction significantly increases the results.

In the table 3, results are significantly enhanced by utilizing of the proposed filter on the Barbara watermarked image in compare with normal extraction. As it shown in this table, proposed method show less improvement for the average frequency image, however, the improvement still is meaningful in compare with normal results. Using unsharp mask before recovery algorithm is more successful in salt and pepper noise and blurring attacks. However, in the jpeg compression and scaling attack, using unsharp mask improve the results. 
Table 2. Comparison in term of NC between the recovered watermarks from the peppers watermarked image with different attack.

\begin{tabular}{|c|c|c|c|c|c|c|}
\hline \multirow[b]{2}{*}{$\begin{array}{l}\text { Pepper } \\
\text { Image }\end{array}$} & \multirow[b]{2}{*}{ PSNR } & \multicolumn{5}{|c|}{ Attack } \\
\hline & & $\begin{array}{l}\text { Attack } \\
\text { Free }\end{array}$ & $\begin{array}{c}\text { Jpeg } \\
\text { compression } \\
(50 \%)\end{array}$ & $\begin{array}{c}\text { Scaling } \\
(50 \%)\end{array}$ & $\begin{array}{c}\text { Salt and } \\
\text { Pepper } \\
\text { Noise } \\
(20 \%)\end{array}$ & $\begin{array}{l}\text { Blurring } \\
\text { (Gaussian } \\
\text { with } \mathrm{h}=5 \text {, } \\
\text { and } \sigma=1 \text { ) }\end{array}$ \\
\hline \multirow{3}{*}{$\begin{array}{l}\text { Without } \\
\text { Filtering }\end{array}$} & 30 & 0.9815 & 0.9284 & 0.7950 & 0.7573 & 0.8478 \\
\hline & 35 & 0.9430 & 0.7996 & 0.7218 & 0.6681 & 0.7712 \\
\hline & 40 & 0.9049 & 0.7022 & 0.6758 & 0.6257 & 0.7186 \\
\hline \multirow{3}{*}{$\begin{array}{l}\text { Convolving } \\
\text { UnSharp } \\
\text { mask before } \\
\text { Extraction }\end{array}$} & 30 & 0.9991 & 0.9746 & 0.8839 & 0.9046 & 0.9234 \\
\hline & 35 & 0.9887 & 0.8522 & 0.8162 & 0.7837 & 0.8692 \\
\hline & 40 & 0.9633 & 0.7373 & 0.7507 & 0.7019 & 0.8132 \\
\hline
\end{tabular}

Table 3. Comparison in term of NC between the extracted watermarks from the Barbara watermarked image with different methods.

\begin{tabular}{l|c|c|c|c|c|c|}
\multirow{2}{*}{$\begin{array}{l}\text { Barbara } \\
\text { Image }\end{array}$} & PSNR & Attack & $\begin{array}{c}\text { Jpeg } \\
\text { compression } \\
(50 \%)\end{array}$ & $\begin{array}{c}\text { Scaling } \\
(50 \%)\end{array}$ & $\begin{array}{c}\text { Salt and } \\
\text { Pepper } \\
\text { Noise } \\
(20 \%)\end{array}$ & $\begin{array}{c}\text { Blurring } \\
\text { (Gaussian } \\
\text { with } \mathrm{h}=5, \\
\text { and } \sigma=1)\end{array}$ \\
\cline { 2 - 7 } & 30 & 0.9493 & 0.8860 & 0.7621 & 0.7624 & 0.8072 \\
\hline \multirow{2}{*}{$\begin{array}{l}\text { Without } \\
\text { Filtering }\end{array}$} & 35 & 0.8906 & 0.7836 & 0.7054 & 0.6853 & 0.7484 \\
\cline { 2 - 7 } & 40 & 0.8153 & 0.6564 & 0.6457 & 0.6258 & 0.6888 \\
\hline $\begin{array}{l}\text { Convolving } \\
\begin{array}{l}\text { UnSharp } \\
\text { mask } \\
\text { before }\end{array}\end{array}$ & 30 & $\mathbf{0 . 9 6 8 1}$ & $\mathbf{0 . 9 3 3 1}$ & $\mathbf{0 . 8 2 7 6}$ & $\mathbf{0 . 8 6 6 0}$ & $\mathbf{0 . 8 7 0 5}$ \\
\cline { 2 - 7 } \begin{tabular}{l} 
Extraction \\
\cline { 2 - 7 }
\end{tabular} & 40 & $\mathbf{0 . 9 4 0 4}$ & $\mathbf{0 . 8 3 2 5}$ & $\mathbf{0 . 7 6 1 4}$ & $\mathbf{0 . 7 7 2 1}$ & $\mathbf{0 . 8 0 9 5}$ \\
\hline
\end{tabular}


Finally, the results of convolving unsharp mask on Baboon image as a representation of image with high spatial frequency are shown in the table 4 . The results are considerably augmented by convolving of the Unsharp Mask with the watermarked image in compare with standard recovery. Convolving proposed filter before retrieval algorithms is more effective in case of salt and pepper noise and blurring attacks and to somewhat in the scaling and jpeg compression attack. Therefore, for high spatial frequency, convolving the unsharp mask before running recovery could augment the results.

Table 4. Comparison in term of NC between the extracted watermarks from the Baboon watermarked image with different methods.

\begin{tabular}{c|c|c|c|c|c|c|}
\multirow{2}{*}{$\begin{array}{c}\text { Baboon } \\
\text { Image }\end{array}$} & PSNR & \multicolumn{5}{|c|}{ Attack } \\
\cline { 3 - 7 } & $\begin{array}{c}\text { Attack } \\
\text { Free }\end{array}$ & $\begin{array}{c}\text { Jpeg } \\
\text { compression } \\
(50 \%)\end{array}$ & $\begin{array}{c}\text { Scaling } \\
(50 \%)\end{array}$ & $\begin{array}{c}\text { Salt and } \\
\text { Pepper } \\
\text { Noise } \\
(20 \%)\end{array}$ & $\begin{array}{c}\text { Blurring } \\
\text { (Gaussian } \\
\text { With } \mathrm{h}=5, \\
\text { and } \sigma=1)\end{array}$ \\
\hline \multirow{3}{*}{$\begin{array}{c}\text { Without } \\
\text { Filtering }\end{array}$} & 35 & 0.9005 & 0.8100 & 0.6456 & 0.6930 & 0.7062 \\
\cline { 2 - 7 } & 40 & 0.8094 & 0.7009 & 0.5896 & 0.6312 & 0.6357 \\
\hline $\begin{array}{c}\text { Convolving } \\
\text { UnSharp } \\
\text { mask } \\
\text { before }\end{array}$ & 30 & $\mathbf{0 . 9 9 0 3}$ & $\mathbf{0 . 9 5 2 0}$ & $\mathbf{0 . 7 7 1 2}$ & $\mathbf{0 . 9 0 9 0}$ & $\mathbf{0 . 8 7 3 7}$ \\
\cline { 2 - 7 } \begin{tabular}{c} 
Extraction \\
\cline { 2 - 7 }
\end{tabular} & 40 & $\mathbf{0 . 9 3 9 9}$ & $\mathbf{0 . 8 5 0 5}$ & $\mathbf{0 . 6 8 7 6}$ & $\mathbf{0 . 8 0 2 0}$ & $\mathbf{0 . 7 8 7 7}$ \\
\hline
\end{tabular}

\section{Conclusions}

In this paper, a new application of Unsharp Mask filter is presented. This filter is used to increase the quality of recovered watermark in the spatial domain watermarking algorithms. The Unsharp Mask filter is convolved with watermarked image before running retrieval algorithm. In this paper we proved that the quality of extracted watermark could increase by applying this filter. Several tests are done to investigate efficiency of this filter on spatial domain watermarking method. Efficiency of the methods is tested by comparing its results with normal recovery method in the term of NC. The watermark is extracted after common image processing attacks with higher NC value by using Unsharp Mask filter. Especially, performance is increased better in case of blurring filter, jpeg compression and scaling. Therefore, it is suggested to use Unsharp Mask filter before running watermark retrieval algorithms in the spatial domain watermarking method.

\section{ACKNOWLEDGEMENTS}


This paper is extracted from research projects for Islamic Azad University, Ramsar Branch, Iran.

\section{References}

Amirgholipour, S. K., \&Naghsh-Nilchi, A. R.. "Robust Digital Image Watermarking Based on Joint DWTDCT". JDCTA, 3(2), (2009), pp. 42-54 .

[2] Kasmani, S. A., \&Naghsh-Nilchi, A., "A new robust digital image watermarking technique based on joint DWT-DCT transformation". In Convergence and Hybrid Information Technology, (2008). ICCIT'08. Third International Conference on (Vol. 2, pp. 539-544). IEEE .

[3] Kasmani, S. A., Mahfouzi, M., \&Asfia, M., " A New Pre-processing Approach to Improve DCT-Based Watermarkings Extraction". In Computer Science and Information Technology-Spring Conference, (2009). IACSITSC'09. International Association of (pp. 131-135). IEEE .

[4] Potdar, V. M., Han, S., \& Chang, E.." A survey of digital image watermarking techniques. In Industrial Informatics, 2005. INDIN'05. (2005) 3rd IEEE International Conference on (pp. 709-716). IEEE .

[5] Kasmani, Saeed Amirgholipour, and Aboosaleh Mohammad Sharifi., "A Pre-Filtering Method to Improve Watermark Detection Rate in DCT based Watermarking.", International Arab Journal of Information Technology (IAJT), March, Vol 11, No., (2014), pp. 178:185.

[6] Depovere, G., Kalker, T., \&Linnartz, J. P. "Improved watermark detection reliability using filtering before correlation. In Image Processing, (1998). ICIP 98. Proceedings. 1998 International Conference on (Vol. 1, pp. 430-434). IEEE .

[7] Chu, W. C. "DCT-based image watermarking using subsampling". Multimedia, IEEE Transactions on, 5(1), (2003), pp. 34-38.

[8] Lin, S. D., \& Chen, C. F.," A robust DCT-based watermarking for copyright protection". Consumer Electronics, IEEE Transactions on, 46(3), (2000), pp. 415-421.

[9] Hsieh, M. S., Tseng, D. C., \& Huang, Y. H.." Hiding digital watermarks using multiresolution wavelet transform". Industrial Electronics, IEEE Transactions on, 48(5), (2001), pp. 875-882 .

[10] Reddy, A. A., \&Chatterji, B. N. "A new wavelet based logo-watermarking scheme". Pattern Recognition Letters, 26(7), (2005), pp. 1019-1027.

[11] Fotopoulos, V., \&Skodras, A. N., "Improved watermark detection based on similarity diagrams". Signal Processing: Image Communication, 17(4), (2002), pp. 337-345 .

[12] Braudaway, G. W., \&Mintzer, F. C. "Application of blurring filters to improve detection of invisible image watermarks". In Proceedings of SPIE (Vol. 5020, (2003), pp. 269-277).

[13] Malik, H., Khokhar, A., \& Ansari, R, “Improved watermark detection for spread-spectrum based watermarking using independent component analysis". In Proceedings of the 5th ACM workshop on Digital rights management, (2005), pp. 102-111. 
[14] Pan, Z., Li, L., Zhang, M., \& Zhang, D., “ Watermark extraction by magnifying noise and applying global minimum decoder" . In Image and Graphics, (2004). Proceedings. Third International Conference on (pp. 349-352). IEEE .

[15] Gonzalez, R. C., \& Woods, R. E, “Digital Image Processing: Introduction”, (2004), pp:187-191.

[16] Polesel, Andrea, Giovanni Ramponi, and V. John Mathews. "Adaptive unsharp masking for contrast enhancement." In Image Processing, (1997). Proceedings., International Conference on, vol. 1, pp. 267270. IEEE. 